\title{
Microfluidic Enabled Portable ABO Reverse Typing Sensor ${ }^{\dagger}$
}

\author{
Shadi Karimi 1,*, Allinson Zuñiga ${ }^{2}$, Pouya Mehrdel ${ }^{1}$, Josep Farre Lladós ${ }^{1}$, Teresa Vidal ${ }^{2}$, \\ Maria Blanca Roncero ${ }^{2}$ and Jasmina Casals Terré 1 * \\ 1 Department of Mechanical Engineering, MicroTech Laboratory, Terrassa 08222, Spain; \\ Pouya.mehrdel@upc.edu (P.M.); Josep.farre.llados@upc.edu (J.F.L.) \\ 2 CELBIOTECH Paper Engineering Research Group, Universitat Politecnica de Catalunya, Terrassa 08222, \\ Spain; Allinson.zuniga@estudiant.upc.edu (A.Z.); Teresa.vidal@upc.edu (T.V.); \\ Blanca.roncero@upc.edu (M.B.R.) \\ * Correspondence: shadi.karimi@upc.edu (S.K.); Jasmina.Casals@upc.edu (J.C.T.) \\ + Presented at the 5th International Symposium on Sensor Science (I3S 2017), Barcelona, Spain, \\ 27-29 September 2017.
}

Published: 6 December 2017

Blood typing is especially important in any emergency transfusion and during pregnancy. Transfusion of a non-compatible blood type can carry out severe health problems, since hemoagglutation reaction with opposite antibodies stick cells together.

Conventional blood typing test in most countries require direct and reverse blood typing. The second step, called reverse typing is done using the natural antibodies from the patient's plasma. The plasma of blood without cells is mixed with blood that is known to be type A and type B. Persons with type A blood have anti-B antibodies, and those with type B blood have anti-A antibodies. Type O blood contains both types of antibodies. The agglutination of the aforementioned cells will allow the determination of the blood type from the natural antibodies of patient's plasma.

Currently, this step is done in clinic labs since it requires a centrifuge to separate plasma from blood. This study presents an approach that combines a microfluidic a blood plasma separator and a paper-based blood type detector.

The proposed high throughput blood plasma separator device has been designed to use cross flow filtration in order to extract higher volume of plasma $(0.1 \mu \mathrm{L})$ from fresh undiluted blood $(2 \mu \mathrm{L})$ with high purity $(100 \%)$ in an admissible time $(5 \mathrm{~min})$ to implement for blood typing tests.

The main advantage of this design is the efficiency and maximization of the amount of obtained plasma from initial sample. The results of this microfluidic system have been compared with commercial lateral flow assays to validate the quality and quantity of the extracted plasma and to prove its capability as a blood back typing input.

Acknowledgement: The research for this article was financially supported by the Spanish Ministry of Economy and Competitiveness, grant no. CTQ2013-48995-C2-1-R.

(C) 2017 by the authors. Licensee MDPI, Basel, Switzerland. This article is an open access article distributed under the terms and conditions of the Creative Commons Attribution (CC BY) license (http://creativecommons.org/licenses/by/4.0/). 